\title{
Silicon-on-Insulator polarization rotating micro-ring resonator
}

\author{
D. Vermeulen, P. De Heyn, T. Van Vaerenbergh, W. Bogaerts, G. Roelkens \\ Photonics Research Group, Department of Information Technology, \\ Ghent University - imec, B-9000 Ghent, Belgium
}

\begin{abstract}
We propose a novel micro-ring resonator which uses quasi-TE polarized light in the bus waveguide to excite the quasi-TM polarized modes in a micro-ring. An all-pass filter is demonstrated on Silicon-on-Insulator.
\end{abstract}

\section{Introduction}

Resonant devices are considered to be a key technology in integrated photonic circuits. For high index contrast platforms such as Silicon-on-Insulator (SOI) the most investigated class of components are definitely the micro-ring resonators [ref]. They can be used as spectral filters for telecommunication and data communication purposes, as wavelength dependent electro-optic modulators and photodetectors, as transducers for label-free biosensing [1], as optical delay lines, ... In general quasi-TE polarized light is used in micro-ring resonators since it has the strongest confinement which enables light guiding in bends with very small radii with negligible bending losses. However, depending on the application and exact platform dimensions, the fundamental quasi-TM polarized mode can be preferred to resonate in the micro-ring. The lower confinement of the quasi-TM mode can be for example beneficial for a-thermal devices [2], sensors based on a cladding refractive index variation, high-Q rings due to a lower backscattering from the vertical sidewalls [3] and for large wavelength range tuning using liquid crystals [4]. Most of these applications, however, exhibit waveguides which are vertically asymmetrical.

In [5] we showed how an asymmetrical waveguide is a major problem for a grating coupler enabled platform. During the adiabatic transition from a broad multimode waveguide to a single-mode waveguide, several anti-crossings will be passed, thereby coupling the fundamental quasi-TM mode to higher order quasi-TE modes which are not guided when tapering down to a single-mode photonic wire waveguide. Circumvention of such an adiabatic taper has been demonstrated by using focusing techniques such as a focusing grating coupler, but with a lower coupling efficiency than similar grating couplers for quasi-TE polarized light. Secondly, for routing purposes one prefers the polarization which enables the most compact routing functionality, i.e. the TE polarization.

A straightforward solution would be to use a cascade of polarization rotators [6], polarization filters and ring-resonator. As a solution we propose a novel micro-ring resonator which uses quasi-TE polarized light in the bus waveguide to excite the quasi-TM polarized modes in a micro-ring, thus acting as a polarization rotator, polarization filter and ring resonator at the same time.

\section{Design}

The coupling section of the ring resonator is an asymmetrical directional coupler which acts as a polarization rotator $[7,8]$. For a certain width of the bus waveguide, the quasi-TE mode in the bus waveguide will be phase matched to the quasi-TM mode in the micro-ring. In order to achieve successful coupling between these two quasi-polarized modes, a vertical asymmetry is necessary [5].

Our design is based on an SOI platform exhibiting a $220 \mathrm{~nm}$ thick Si core layer on a $2 \mu \mathrm{m}$ thick buried oxide (BOX) layer. The asymmetry requirement is fulfilled by using an air top cladding. A deep etch of $220 \mathrm{~nm}$ is performed for defining the waveguides and ring resonator. A shallow etch of $70 \mathrm{~nm}$ allows us to use grating couplers for characterization. We choose a bus waveguide width of $320 \mathrm{~nm}$ and a micro-ring waveguide width of $600 \mathrm{~nm}$. The gap of the coupling section is $300 \mathrm{~nm}$ and the ring radius is $30 \mu \mathrm{m}$. The bus waveguide has the same curvature as the micro-ring, resulting in a plane symmetric coupling section.

\section{Measurements}

The measurement result is shown in Figure 1. Because the grating couplers act as a coupling method for TE-polarized light and as polarization filter with an extinction ratio of $50 \mathrm{~dB}$, we know that only the quasi-TE mode is excited in the bus waveguide. By calculating the theoretically expected Free Spectral Range (FSR) of the quasi-TM resonant mode 


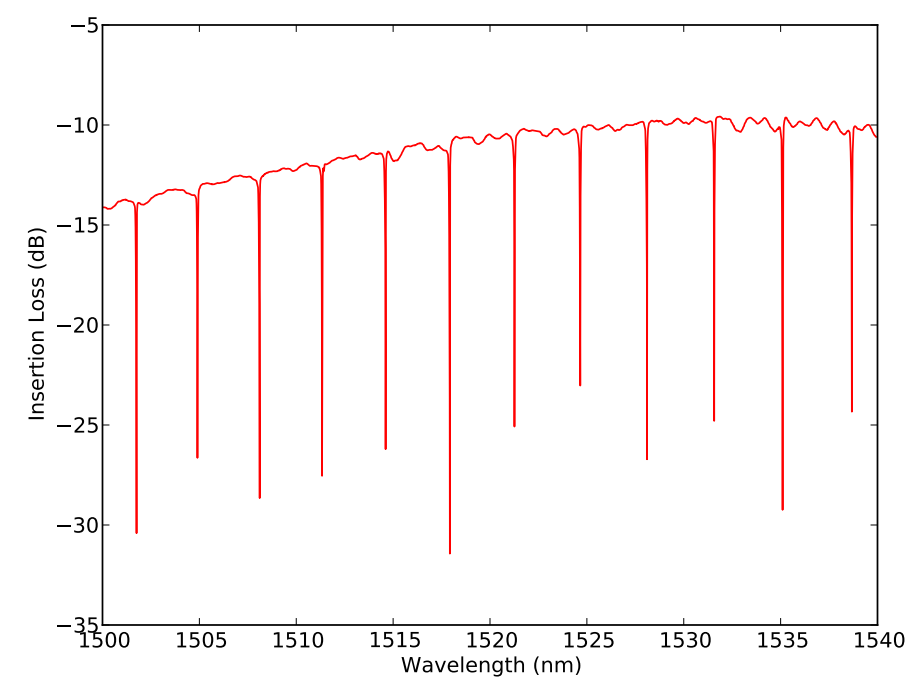

Fig. 1: Measurement result of the polarization rotating micro-ring resonator in an all-pass filter configuration.

and comparing it with the measured FSR, we can conclude that quasi-TE polarized light from the bus waveguide is coupled to the quasi-TM resonant mode in the ring. The mean extinction ratio (ER) over the $40 \mathrm{~nm}$ wavelength range is $-16.2 \mathrm{~dB}$, which means that the ring is critically coupled. The mean $Q$ is 32660 and the mean finesse is 88 . Furthermore, no peak splitting was detected.

\section{Conclusion}

We proposed a new kind of micro-ring resonator which makes use of a polarization rotating directional coupling section to excite the quasi-TM resonant mode. A critically coupled all-pass filter showing very good performance was demonstrated. This polarization micro-ring resonator is expected to be useful for a whole class of applications where TM-polarized light is preferred to resonate.

\section{Acknowledgements}

Diedrik Vermeulen thanks the Institute for the Promotion of Innovation by Science and Technology in Flanders (IWT) for a grant. Thomas Van Vaerenbergh is supported by the Flemish Research Foundation (FWO-Vlaanderen) for a PhD grant.

\section{References and links}

1. K. De Vos, I. Bartolozzi, E. Schacht, P. Bienstman, and R. Baets, "Silicon-on-Insulator microring resonator for sensitive and label-free biosensing." Optics express, vol. 15, no. 12, pp. 7610-5, Jun. 2007. [Online]. Available: http://www.ncbi.nlm.nih.gov/pubmed/19547087

2. J. Teng, P. Dumon, W. Bogaerts, H. Zhang, X. Jian, X. Han, M. Zhao, G. Morthier, and R. Baets, "Athermal Silicon-on-insulator ring resonators by overlaying a polymer cladding on narrowed waveguides." Optics express, vol. 17, no. 17, pp. 14 627-33, Aug. 2009. [Online]. Available: http://www.ncbi.nlm.nih.gov/pubmed/19687941

3. P. D. Heyn, B. Kuyken, D. Vermeulen, W. Bogaerts, and D. V. Thourhout, "High-Performance Low-Loss Silicon-on-Insulator Microring Resonators using TM-polarized Light," in Optical Fiber Communication Conference. Optical Society of America, 2011.

4. W. D. Cort, J. Beeckman, R. James, F. A. Fernandez, R. Baets, and K. Neyts, "Tuning silicon-on-insulator ring resonators with in-plane switching liquid crystals," JOSA B, vol. 28, no. 1, pp. 79-85, 2011.

5. D. Vermeulen, K. V. Acoleyen, S. Ghosh, S. Selvaraja, W. A. D. D. Cort, N. A. Yebo, E. Hallynck, K. D. Vos, P. P. P. Debackere, P. Dumon, W. Bogaerts, G. Roelkens, D. V. Thourhout, and R. Baets, "Efficient Tapering to the Fundamental Quasi-TM Mode in Asymmetrical Waveguides," in European Conference on Integrated Optics, 2010.

6. D. Vermeulen, S. Selvaraja, P. Verheyen, W. Bogaerts, D. V. Thourhout, and G. Roelkens, "High Efficiency Broadband Polarization Rotator on," in Group IV Photonics, 2010, pp. 42-44. [Online]. Available: http://ieeexplore.ieee.org/xpl/freeabs_all.jsp?arnumber=5643432\&abstractAccess=no\&userType=inst

7. L. Liu, Y. Ding, K. Yvind, and J. r. M. Hvam, "Silicon-on-insulator polarization splitting and rotating device for polarization diversity circuits," Optics Express, vol. 19, no. 13, pp. 12 646-12 651, 2011.

8. D. Dai and J. E. Bowers, "Novel concept for ultracompact polarization splitter-rotator based on silicon nanowires." Optics express, vol. 19, no. 11, pp. 10 940-9, May 2011. [Online]. Available: http://www.ncbi.nlm.nih.gov/pubmed/21643354 